\title{
Triggering on jets in heavy ion collisions at the ATLAS experiment
}

\section{Martin Rybar for the ATLAS Collaboration*}

Charles University in Prague, Faculty of Mathematics and Physics

E-mail: rybar@ipnp.troja.mff.cuni.cz

The ATLAS detector is designed primarily for proton-proton collisions, and potentially interesting events are selected by a three-level trigger system. We have studied the performance of the ATLAS trigger system for $\mathrm{Pb}+\mathrm{Pb}$ collisions. First, we describe the ATLAS trigger system and triggering strategy. We then focus on the first level calorimeter trigger and propose modest changes to the default trigger strategy to address specific characteristics of heavy ion collisions at the LHC.

High-pT Physics at LHC -09

February 4- 42009

Prague, Czech Republic

\footnotetext{
* Speaker.
} 


\section{Introduction}

The ATLAS detector, shown in Fig.1, is one of two general purpose detectors at the Large Hadron Collider (LHC) which are designed for $\mathrm{p}+\mathrm{p}$ collisions at $14 \mathrm{TeV}$ centre of mass energy and built to perform precision measurements of the Standard Model parameters, to search for the Higgs boson and to search for Supersymmetry and other physics beyond Standard Model. The bunchcrossing frequency of $\mathrm{p}+\mathrm{p}$ collisions is $40 \mathrm{MHz}$, leading to an interaction rate of approximately $1 \mathrm{GHz}$ at the nominal luminosity $10^{34} \mathrm{~cm}^{-2} \mathrm{~s}^{-1}$. Therefore the data flow is in an order of $10^{7}$ $\mathrm{MBs}^{-1}$. Currently, this rate is too large for the expected archiving system. The solution is to have a trigger system which selects $200 \mathrm{~Hz}$ of potentially interesting events, reducing the data flow to approximately $300 \mathrm{MB} / \mathrm{s}$ for archiving.

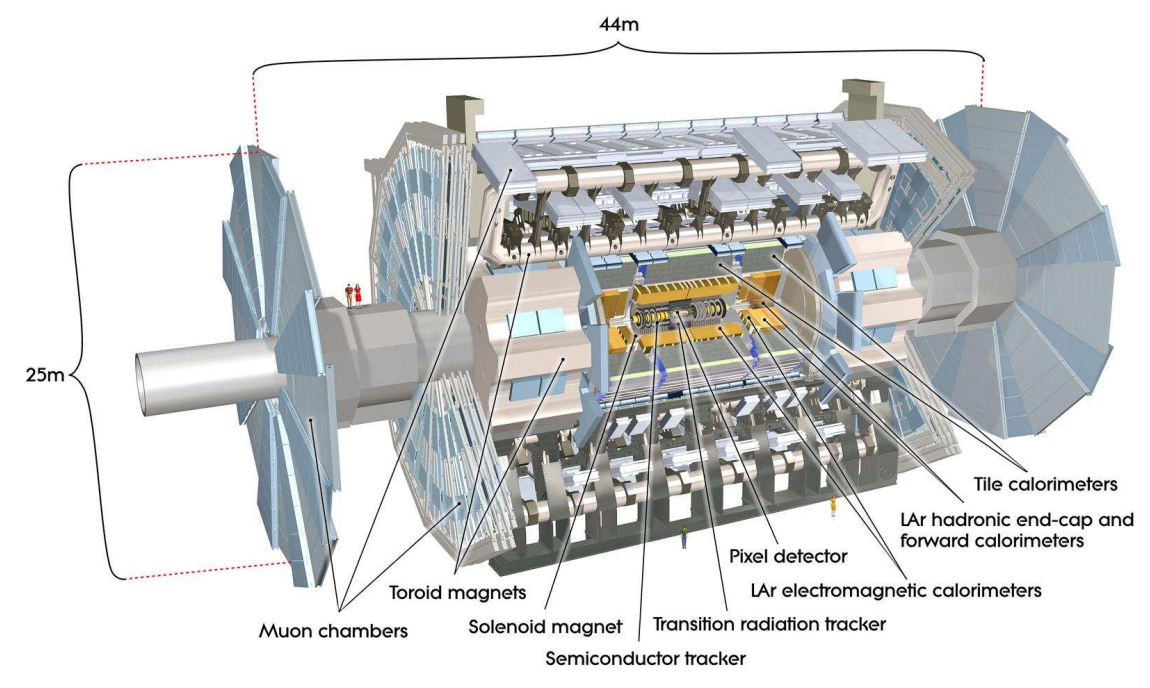

Figure 1: The ATLAS detector.

Since $\mathrm{p}+\mathrm{p}$ and $\mathrm{Pb}+\mathrm{Pb}$ collisions differ significantly in event rates and event size (see table 1.) it is clear that some modifications need to be made to the default trigger configuration.

\begin{tabular}{|c|c|c|}
\hline & $\mathrm{p}+\mathrm{p}$ & $\mathrm{Pb}+\mathrm{Pb}$ \\
\hline High designed luminosity & $10^{34} \mathrm{~cm}^{-2} \mathrm{~s}^{-1}$ & $10^{27} \mathrm{~cm}^{-2} \mathrm{~s}^{-1}$ \\
\hline Charged particles multiplicity & 200 & 3000 \\
\hline Interaction rate & $1 \mathrm{GHz}$ & $7.7 \mathrm{kHz}$ \\
\hline Event size & $1.5 \mathrm{Mb}$ & $5 \mathrm{Mb}$ \\
\hline Maximum archiving rate & $200 \mathrm{~Hz}$ & $60 \mathrm{~Hz}$ \\
\hline
\end{tabular}

Table 1: Comparison between expectations for $\mathrm{p}+\mathrm{p}$ [1] and $\mathrm{Pb}+\mathrm{Pb}$ collisions [2]

\section{ATLAS trigger system}

To achieve the desired reduction in trigger rate, ATLAS has a three-level trigger system. The 
First-Level Trigger (L1) is hardware based. The High-Level Trigger (HLT) is primarily implemented in software and is subdivided into Level-2 (L2) and the Event Filter (EF). An overview of the trigger system is shown in Fig. 2. After an event is accepted by the L1, the data from pipeline memories are transferred through the derandomizer to the read out Driver (ROD) out of the detector. The ROD is an interface to the data acquisition (DAQ) system that defines the data format of the event and provides error detection and recovery mechanisms. The data waiting for the L2 decision are temporarily stored in the read out buffers (ROB). Events accepted by L2 are transferred to the event-building system and then to EF for a final selection.

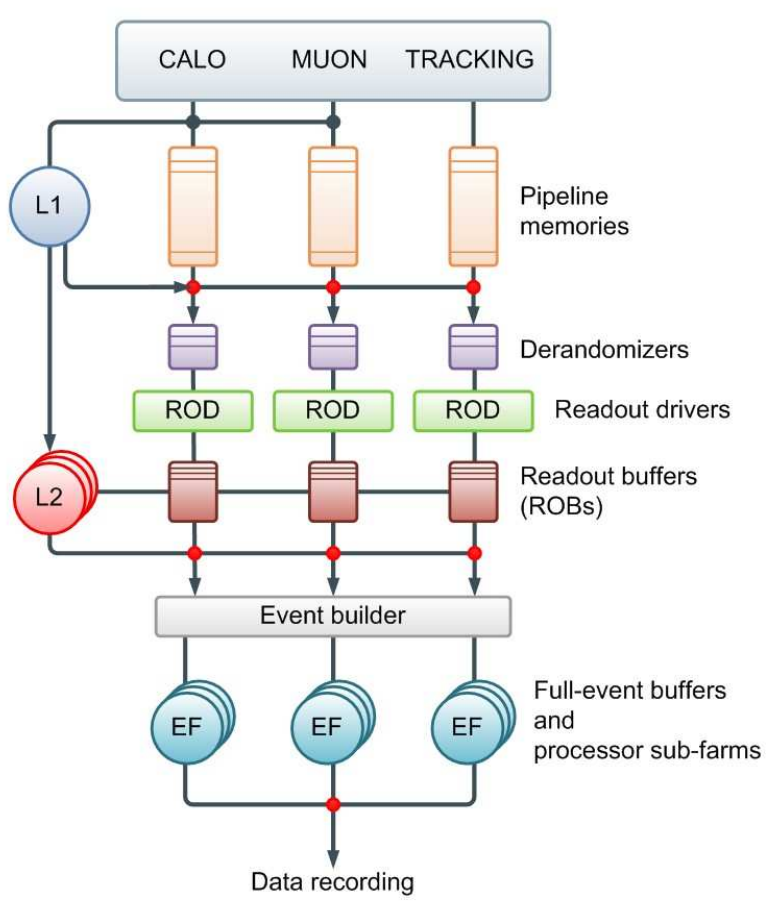

Figure 2: Overview of the ATLAS trigger system.

\subsection{Level-1 trigger}

The L1 trigger is a hardware-based system which can use only simple algorithms because of its small latency limit of $2.5 \mu \mathrm{s}$. It uses only a subset of the ATLAS detector, particularly the calorimeters and muon chambers with reduced granularity. The goal of L1 is to reduce the event rate to $75 \mathrm{kHz}$. However, since the maximum interaction rate of $\mathrm{Pb}+\mathrm{Pb}$ collisions is $7.7 \mathrm{kHz}$ [2] a rejection at L1 is not needed. Despite this fact we need an efficient L1 trigger because it provides region of interest (RoI) that are supplied to the level-2 trigger. Events that pass the L1 selection conditions are transferred from the detector-specific front-end electronics to the data acquisition system. 
The basic quantity for the calorimeter trigger is the "trigger tower", an example of which is shown in Fig. 3, which combines cells of the actual calorimeter.

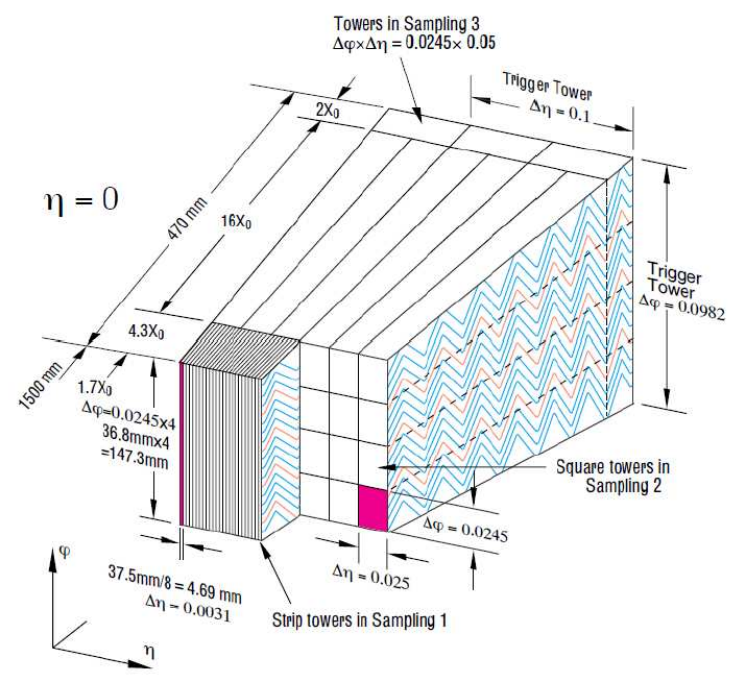

Figure 3: Schematic diagram of the ATLAS EM calorimeter.

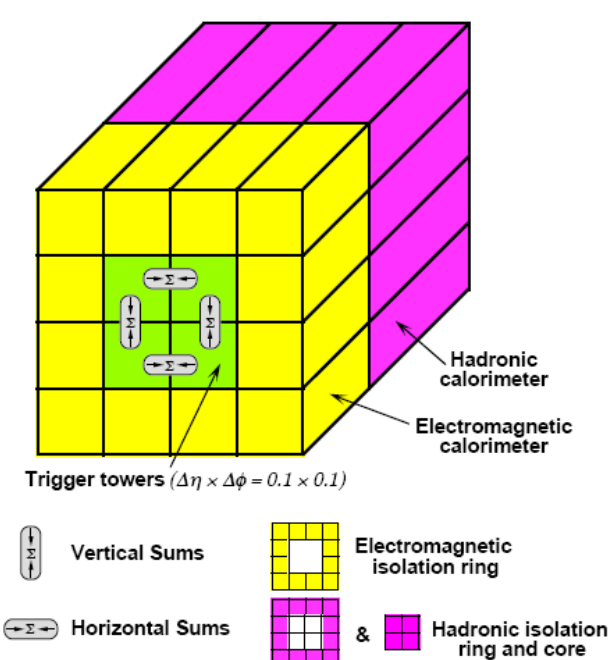

Figure 4: Description of the ATLAS trigger tower scheme for EM/Tau RoI's.

L1 creates the following types of trigger objects:

- EM/Tau RoI: electrons, $\gamma, \tau$ and single hadron candidates. These objects are reconstructed by the electromagnetic calorimeter (electron, photon) and hadronic calorimeter ( $\tau$ and hadrons). The EM/Tau RoI's can be restricted by setting up to 16 thresholds. These thresholds provide criteria for electrons and $\gamma$ hadronic veto and for EM/Tau isolation. The electron/photon and $\tau$ /hadron finding algorithms cover $|\eta|<2.5$ and are based on a sliding window of $4 \times 4$ trigger towers with granularity $0.1 \times 0.1$ in $\eta$ and $\varphi$ (see Fig. 4). Algorithms use six basic elements [4]:

1. Four overlapping EM clusters, each summed over two electromagnetic towers. This element is used to measure the $E_{T}$ of electromagnetic showers.

2. A hadronic core, four hadronic towers centred in the algorithm window behind the EM clusters. This sum is used for isolation tests in the hadronic calorimeters.

3. Four hadronic clusters, each summed in depth over the electromagnetic and hadronic calorimeters. These four hadronic clusters are formed by individually summing twotower EM cluster (item 1) with the hadronic core (item 2). This element is used to measure the $E_{T}$ of hadronic showers.

4. An electromagnetic isolation ring which consists of 12 electromagnetic towers surrounding clusters. This sum is used for isolation tests in the electromagnetic calorimeters.

5. A hadronic isolation ring which consists of 12 hadronic towers behind the electromagnetic isolation ring. This sum is used for isolation tests in the hadronic calorimeters. 
6. A $2 \times 2$-tower cluster RoI, summed in depth over the electromagnetic cluster regions and hadronic core (item 2). Its center is in a local maximum given by the sliding window algorithm.

The requirements for electron/photon RoIs are:

1. The most energetic of the four clusters in the electromagnetic calorimeter must be greater than one of the 16 thresholds.

2. The cluster RoI must be a local $E_{T}$ maximum compared to its neighbours

3. Isolation requirements - the total $E_{T}$ in the electromagnetic/hadronic isolation ring must be less than the corresponding electromagnetic/hadronic isolation ring threshold.

4. The total $E_{T}$ in the hadronic core must be less than the chosen threshold.

The requirements for Tau/hadron objects:

1. The most energetic of the four clusters in the electromagnetic + had. calorimeter must be greater than a given threshold.

2. The cluster RoI must be a local $E_{T}$ maximum compared to its neighbours

3. Isolation requirements, similar to that used for electron/photon RoIs.

- Jet RoI: hadronic jet candidate reconstructed by both hadronic and electromagnetic calorimeter. There are two sets of thresholds: 8 thresholds for central JET RoI's $(|\eta|<3.2)$ and 4 thresholds for forward JET RoI's. The jet trigger algorithm is similar to the $\tau /$ hadron trigger, but it uses a different granularity and a different cluster size. The basic units are the "jet elements", which are formed by summing over $2 \times 2$ trigger towers, which means that the granularity is $0.2 \times 0.2$. Some jet elements in the forward region $(|\eta|>2.4)$ have a different size which implies that the JET RoI's have also different size, as shown in Fig. 5.
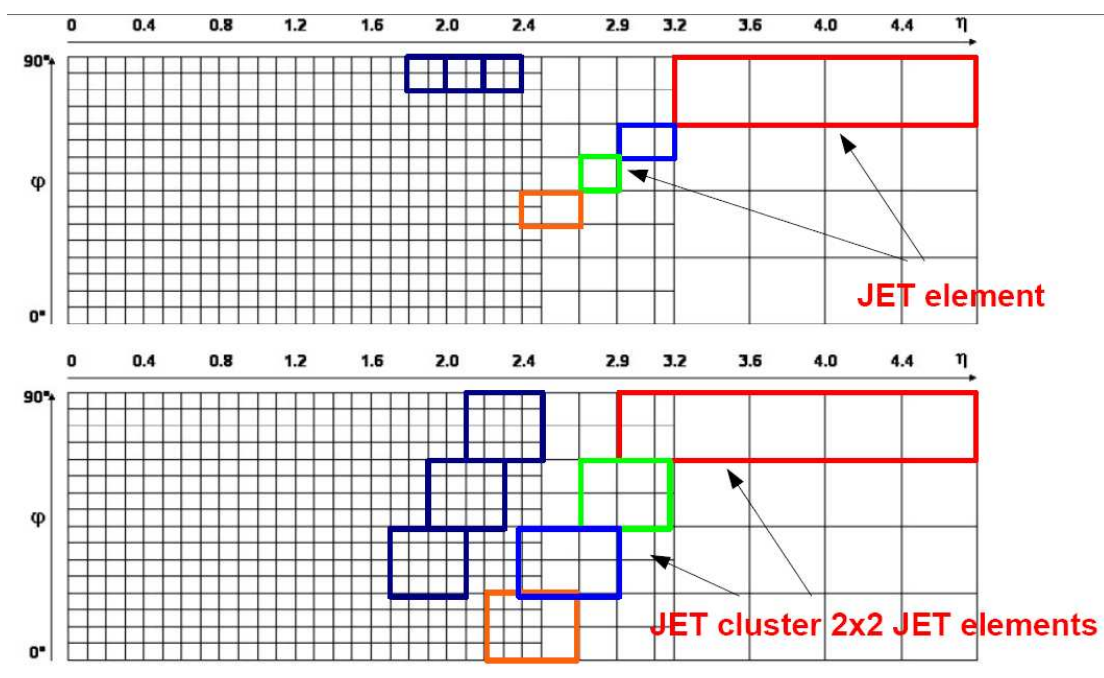

Figure 4: Trigger-tower granularity for $\eta>0$ and one quadrant in $\phi$.

Figure 5: Granularity for triggering in outer region [3]. 
The jet algorithm has two components [4]:

1. A $2 \times 2$ JET RoI

2. Jet windows which are used to measure the jet $E_{T}$. The windows are summed over $2 \times 2,3 \times 3$ or $4 \times 4$ jet elements. The jet window slides in steps of one jet element. We use only the $2 \times 2$ because of the large backgrounds in heavy ion collisions.

There are two requirements for JET RoI's:

1. The cluster RoI must be a local $E_{T}$ maximum compared to its neighbours.

2. The jet window $E_{T}$ must be greater than the jet threshold.

- Total $E_{T}$ : Total transverse energy. Four thresholds are available.

- Missing $E_{T}$ : Signature of neutral weakly interacting particles. A set of 8 thresholds is available.

- Muon RoI: Muon candidate object reconstructed by the ATLAS muon spectrometer. There are 6 independently programmable $\mathrm{p}_{T}$ thresholds available.

There are several other conditions for candidate objects to avoid multiple counting of RoI's. More information about the specifications of the L1 trigger can be found in [1] and [3].

We used PYTHIA [6] events with $70-140 \mathrm{GeV}$ jets and embedded them into unquenchinged HIJING [7] events for testing the L1 trigger system in simulations. We also tested the influence of collision centrality on the trigger by using three samples of different impact parameter, which controls the background multiplicity. The analysis was carried out for three impact parameter selections, $b=2,6,10 \mathrm{fm}$, which have average $\mathrm{d} N / \mathrm{d} \eta=2700,1700,460$.

The trigger efficiency is defined by a number of Rol's that are transferred from L1 to L2. This number is limited by the trigger specifications. The limit for JET RoI's is 32, and the limit for EM/Tau RoI's is 64. If the number of RoI's reaches the limit the recording stops. This implies that loose thresholds can cause an effective loss of a part of calorimeter. An illustration of this effect is in Fig. 6, where the asymmetry in $\eta$ is caused by exceeding the maximum number of RoI's.

The left plot of Fig. 7 shows the number of EM/Tau Rol's for the lowest default p+p thresholds. The right plot of Fig. 7 shows the number of JET Rol's for the lowest default thresholds. It is seen that we need to optimize thresholds in order to accept as many interesting Rol's as possible, but to stay below the maximum allowed. As a first attempt to optmize the L1 trigger, we use dijets to calculate thresholds in order to have an average of five RoI's per event.

Fig. 8 shows EM/Tau and JET RoI's integral distribution for the difference collision centralities. It is evident that the thresholds must depend on centrality, which can be determined using the total $\mathrm{E}_{T}$ trigger information as one can see in Fig. 9. We can require a coincidence in the central trigger processor (CTP) between the total $\mathrm{E}_{T}$ and the threshold for EM/JET RoI's:

$$
\text { total } E_{T}<X \mathrm{TeV} \& \operatorname{RoI} E_{T}>Y \mathrm{GeV}
$$




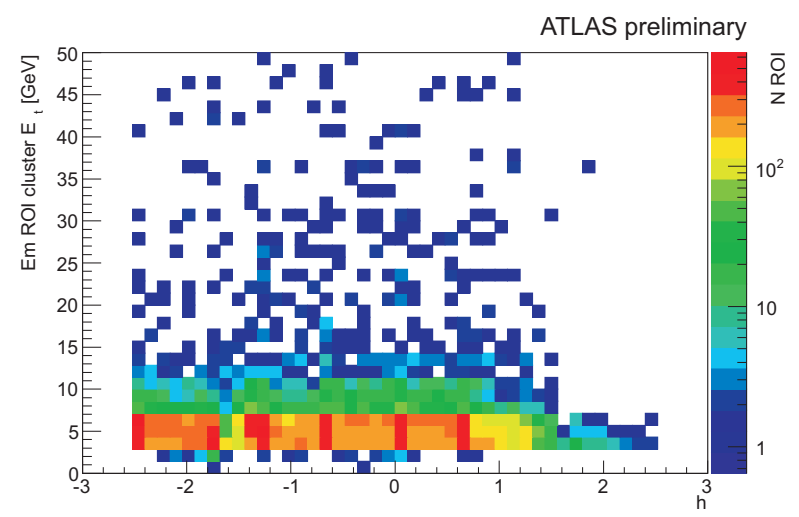

Figure 6: $\mathrm{E}_{T}$ versus $\eta$ distribution of EM/Tau RoI's in electromagnetic calorimeter for centrality $b=6 \mathrm{fm}$.
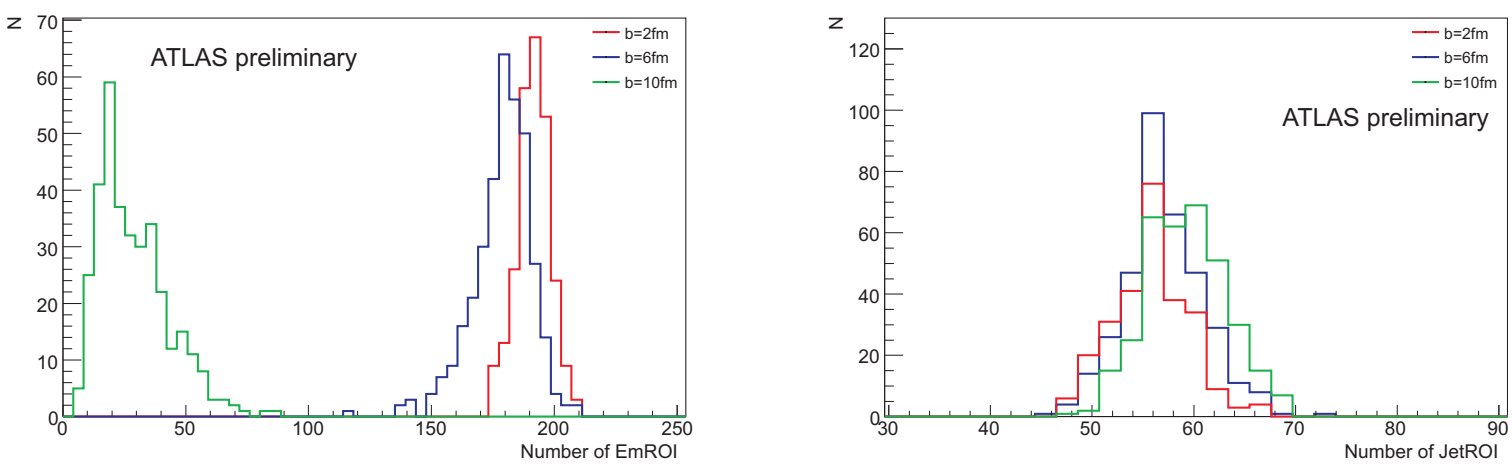

Figure 7: left - Number of EM RoI's for a $3 \mathrm{GeV}$ threshold. right - Number of JET RoI's for a $5 \mathrm{GeV}$ threshold.

We can use predefined thresholds for the EM/Tau and JET RoI's but certainly we will have to redefine the thresholds for total $E_{T}$ because the current values in firmware, designed for $\mathrm{p}+\mathrm{p}$, are too low (the maximum value is presently $650 \mathrm{GeV}$, while the total energy in peripheral collisions is expected to be $2 \mathrm{TeV}$ ). The overall set of suggested thresholds can be found in table 2 .

\begin{tabular}{|c|c|c|}
\hline Total ET [TeV] & JET threshold $[\mathrm{GeV}]$ & EM threshold $[\mathrm{GeV}]$ \\
\hline $0-5$ & 18 & 3 \\
\hline $5-9$ & 35 & 7 \\
\hline $9-13$ & 42 & 7 \\
\hline $13-16$ & 70 & 13 \\
\hline$>16$ & 70 & 13 \\
\hline
\end{tabular}

Table 2: Suggested trigger menu

Fig. 10 shows the distributions of the number of trigger objects for three different centralities for suggested thresholds. One can see that distributions are safely below the saturation limit.

The resulting efficiency can be found in Fig. 11. The efficiency can be further improved by optimizing thresholds for heavy ion collisions (as shown in Table 3) and by running jet-finding 

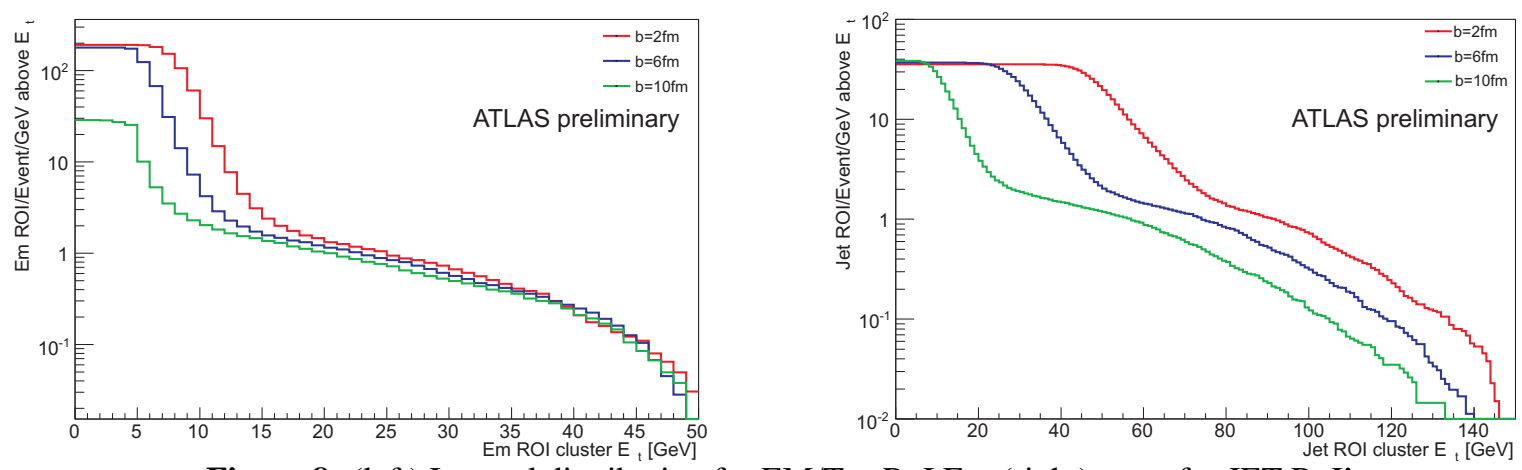

Figure 8: (left) Integral distribution for EM/Tau RoI E ${ }_{T}$, (right) same for JET RoI's.

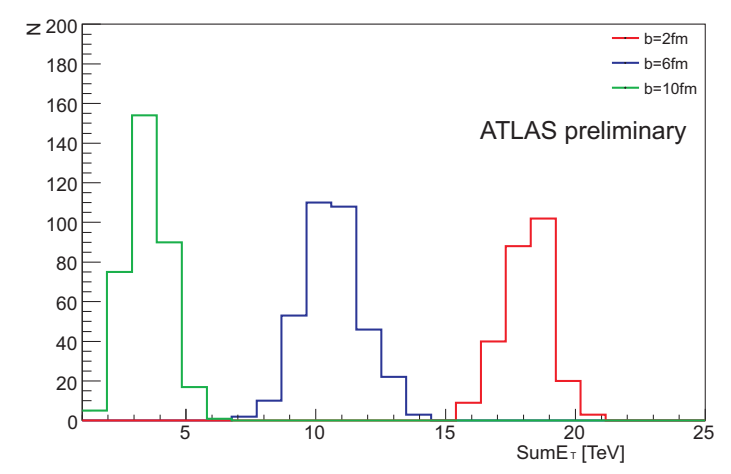

Figure 9: Distribution of total $\mathrm{E}_{T}$ for three centrality bins $(\mathrm{b}=2,6$ and $10 \mathrm{fm})$.
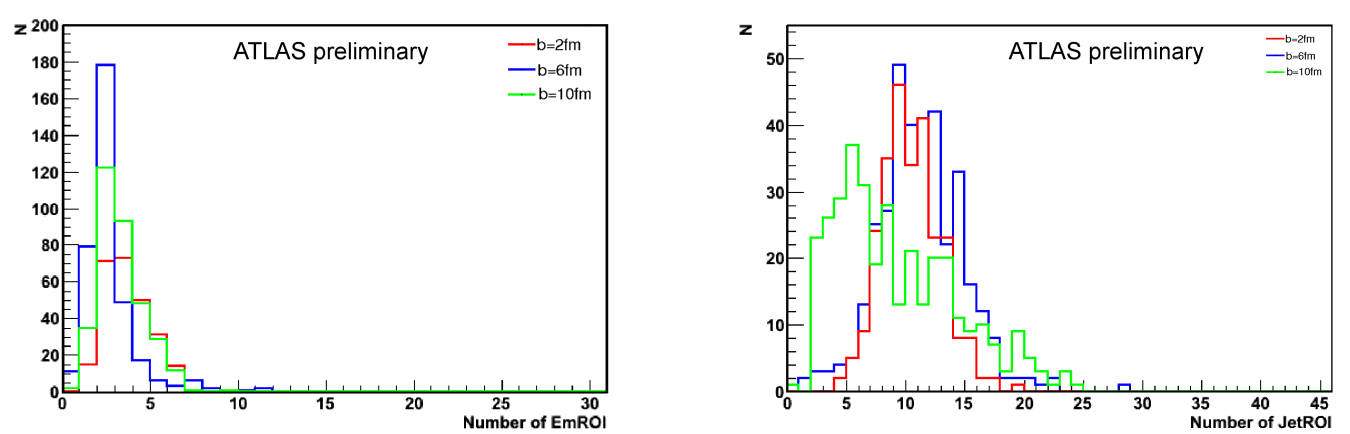

Figure 10: left - Number of EM RoI's with suggested thresholds. right - Number of JET RoI's with suggested thresholds.

algorithms at L2, not only on JET RoI's but also on EM or Tau/hadron Rol's. This can be seen in the right plot of Fig. 12, where Tau/hadron Rol's are also used.

It has been mentioned that the endcaps and forward calorimeters have a coarser granularity, which leads to the RoI's in this region having a larger spread than in the central region (as shown in Fig. 13). This can bias efficiency and increase the number of RolâĂŹs in these regions. An $\eta$ dependent threshold is a partial solution to this problem. We will probably also accept all forward JET Rol's (12 forward JET RoI's in each event) because of their large size (as shown in Fig. 5), making it difficult to estimate if they contain jets at the trigger level. 


\begin{tabular}{|c|c|c|}
\hline Total ET $[\mathrm{TeV}]$ & JET threshold $[\mathrm{GeV}]$ & EM threshold $[\mathrm{GeV}]$ \\
\hline $0-5$ & 18 & 3 \\
\hline $5-9$ & 25 & 6 \\
\hline $9-13$ & 38 & 7 \\
\hline $13-16$ & 50 & 8 \\
\hline$>16$ & 60 & 9 \\
\hline
\end{tabular}

Table 3: Proposed modification of trigger thresholds for ATLAS heavy ion running

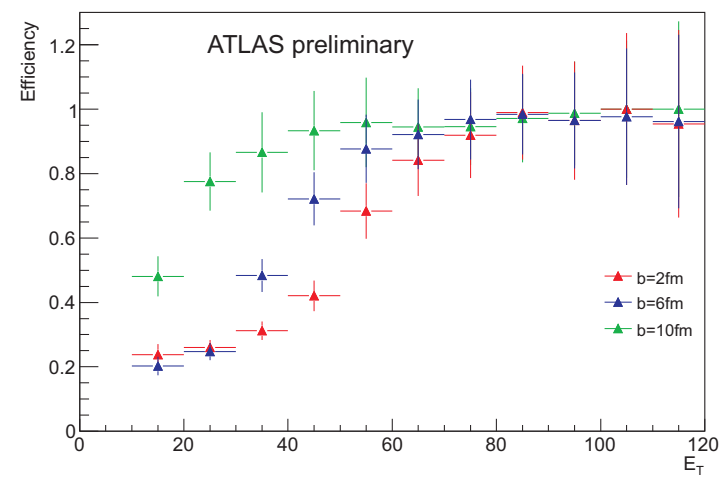

Figure 11: Jet trigger efficiency without any threshold modifications.
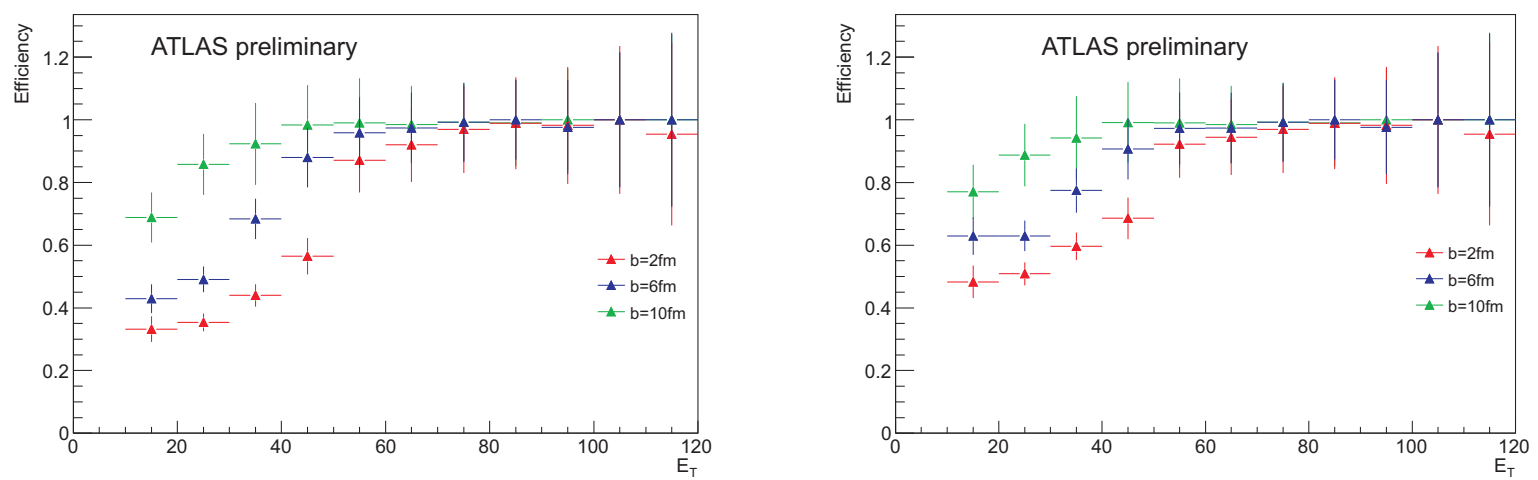

Figure 12: Jet trigger efficiency with modified thresholds and left if Tau/hadron RoI's are included at L2, and right if EM RoI's are included as well.

Overall, it is found that the ATLAS L1 trigger can be used effectively in heavy ion collisions.

\subsection{The Level-2 trigger}

The level-2 (L2) trigger is based on software selection algorithms running in the processor farms to reduce the rate coming from L1 to about $900 \mathrm{~Hz}$. The L2 algorithms are seeded by L1 RoI's so that only a small fraction of the event is transferred to the L2. The L2 can access data from all subdetectors with full granularity and latency limit is $10 \mathrm{~ms}$; thus, it can use more sophisticated algorithms. The L2 trigger refines the decision made by L1 and adds additional selection criteria. 


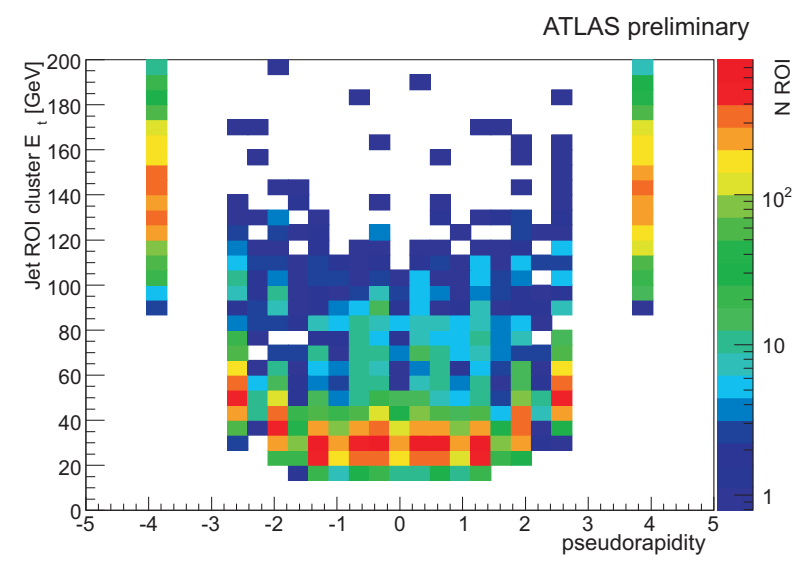

Figure 13: JET's $E_{T}$ versus $\eta$ distribution for centrality $\mathrm{b}=6 \mathrm{fm}$ : strips at large pseudorapidity are forward JET RoI's. The "U-shape" is caused by coarser granularity in the endcaps.

The L2 trigger consists of several steps which are grouped together into a trigger "chain". Each trigger signature (e.g. a $60 \mathrm{GeV}$ jet, or a $20 \mathrm{GeV}$ muon) corresponds to a set of algorithms that are executed sequentially. There are two basic algorithm types:

1. Feature extraction algorithms (FEX) are used to access trigger objects (e.g. clusters, tracks) which are used to create physics objects (e.g. jets, electrons) to confirm the candidate RoI.

2. Hypothesis algorithms are used to reconstruct physics objects and apply cuts to them to reject events.

More information about the L2 trigger can be found in Refs. [1] and [5].

At present, we use the default jet-finding algorithm at L2. It is a simple cone algorithm with three iterations and $R=0.4$. We do not have implementation of any background subtraction yet. Figure 14 shows a first result from the L2 studies: the distribution of jet $E_{T}$ for different centralities. The input data contained jets with parton energy $35-70 \mathrm{GeV}$ and it is clear that the background subtraction is absolutely necessary for any future study, just as it is in the offline analysis.

\subsection{Event filter}

The Event Filter (EF) is also software based. In contrast to L2 it runs after the event building, so it has access to the complete event. Its task is to reduce $\mathrm{Pb}+\mathrm{Pb}$ rate to $60 \mathrm{~Hz}$, with events accepted by the EF archived to mass storage. The EF first refines the L2 decision, and is seeded by L2 similarly to how L2 is seeded by L1 RoIs. However, the EF uses algorithms similar to those used for offline reconstruction, and we expect to adapt our current offline scheme to the trigger in the near future.

\section{Conclusion}

The ATLAS trigger is designed to cope with $\mathrm{p}+\mathrm{p}$ collisions at $1 \mathrm{GHz}$. We have proposed modest changes to adapt it to heavy ion collisions. We have almost $100 \%$ efficiency for jets of 60 


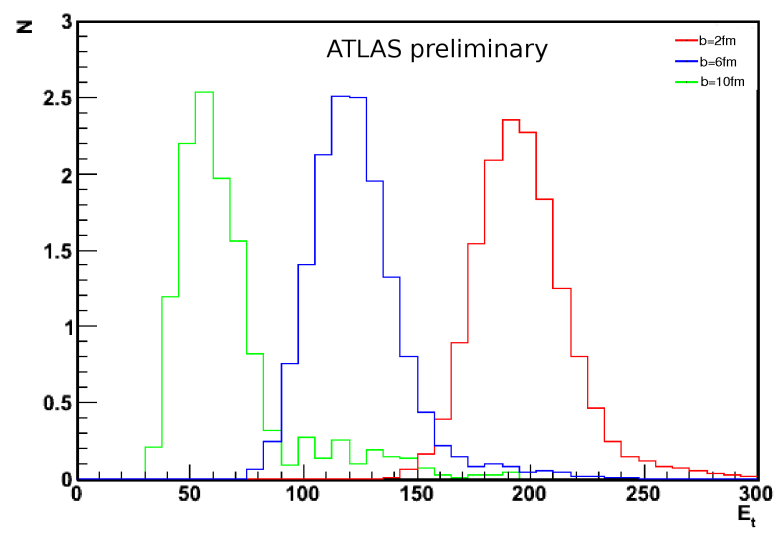

Figure 14: Distribution of jet $\mathrm{E}_{T}$ in L2.

$\mathrm{GeV}$ and $50 \%$ efficiency down to $30 \mathrm{GeV}$ in the most central HIJING events. The proposed L1 trigger menu can be found in Tables 2 and 3. We have studied the performance of the L2 trigger, but not yet done background subtraction. Overall we can conclude that the ATLAS trigger is capable for use in heavy ion collisions.

\section{References}

[1] ATLAS Collaboration, G. Aad et al., The ATLAS experiment at the CERN Large Hadron Collider, 2008, JINST-3-S08003

[2] ATLAS Collaboration, Heavy Ion Physics with the ATLAS Detector, Letter of Intent, CERN/LHC/2004-009, LHCC I-013

[3] ATLAS Collaboration: ATLAS Level-1 Trigger, Technical Design Report, ATLAS TDR-12, CERN, Geneva, 1998

[4] ATLAS L1Calo Group: ATLAS Level-1 Calorimeter Trigger Algorithms, CERN, Geneva, 2008

[5] ATLAS Collaboration: ATLAS High-Level Trigger, Data Acquisition and Controls, Technical Design Report, ATLAS TDR-12, CERN, Geneva, 2002

[6] T. Sjöstrand, S. Mrenna, P. Skands: Pythia 6.4 Physics and Manual, hep-ph/0603175, LU TP 06-13, FERMILAB-PUB-06-052-CD-T, 2006

[7] HIJING: A Monte Carlo model for multiple jet production in $\mathrm{p}+\mathrm{p}, \mathrm{p}+\mathrm{A}$ and $\mathrm{A}+\mathrm{A}$ collisions, $\mathrm{X} . \mathrm{-N}$. Wang and M. Gyulassy, Phys. Rev. D44, 3501 (1991). 